\title{
TERATOGÊNESE GENITURINÁRIA EM GATO MAINE COON
}

\author{
MARCY, Lancia Pereira'; \\ JÉSSICA, Friol'; \\ DENISE, Dias Primo3; \\ CYNTIA, Fernandes ${ }^{4}$;
}

Recebido: 05/05/2017

Aceito: 01/07/2018

${ }^{1}$ Médica Veterinária, Doutora, Professora da UFSC Curitibanos; ${ }^{2}$ Médica Veterinária, Ultrassonografista, Hospital Veterinário Taquaral, Campinas-SP; ${ }^{3}$ Médica Veterinária, Ultrassonografista, Echovet, Campinas-SP; ${ }^{4}$ Médica Veterinária clínica, Clínica Veterinária Cães \& Gatos, Campinas-SP.

\section{RESUMO}

ocorrência de ureteres ectópicos é pouco frequente e mais observada em cadelas,
que apresentam incontinência urinária desde filhotes. Gatos são raramente afetados
por essas duas anormalidades de desenvolvimento. Este relato visa apresentar o caso de um gato macho Maine Coon, de um ano e quatro meses, com diagnóstico de ureteres ectópicos bilaterais, hidronefrose unilateral, além de criptorquidismo unilateral. O paciente foi encaminhado devido à queixa de apatia e hiporexia após orquiectomia unilateral realizada alguns dias antes e não apresentava incontinência urinária. O ureter ectópico e a hidronefrose direitos e testículo ectópico esquerdo foram observados por meio de ultrassonografia, enquanto a ectopia do ureter esquerdo foi observada no período transcirúrgico. Optou-se por fazer nefroureterectomia total direita e orquiectomia esquerda naquele momento, além de debridamento de ferida cirúrgica devido a inúmeros abscessos subcutâneos provenientes da primeira cirurgia realizada. Entretanto, 10 horas pós-cirurgia o paciente teve hipotensão e, mesmo após restabelecimento da pressão arterial, veio a óbito. A tutora não autorizou a realização da necropsia para que se pudesse investigar a causa definitiva da morte.

Palavras-chave: Ectopia. Felino. Hidronefrose. Testículo. Ureter. 


\section{INTRODUÇÃO}

Ectopia ureteral é uma anormalidade congênita do segmento terminal do ureter, pode ser uni ou bilateral. O orifício ureteral pode estar localizado distal ao trígono vesical, desembocando na uretra, vagina, colo da vesícula urinária, ducto deferente, próstata ou outras glândulas sexuais secundárias (MCLOUGHLIN; CHEW, 2000). Esta anomalia é mais comum em cadelas e a inserção vesical do ureter ectópico pode ser intra ou extramural (NEWMAN, 2013). O diagnóstico por imagem é essencial e pode ser realizado através de ultrassonografia, urografia excretora, tomografia computadorizada e cistoscopia (HECHT; HENRY, 2011).

O criptorquidismo caracteriza-se por retenção testicular, com localização em qualquer parte do caminho de descida do testículo, do polo caudal renal até o canal inguinal, ou externo ao canal, mas cranial ao escroto (MEYERS-WALLEN, 2009). Esta desordem de desenvolvimento sexual é relativamente frequente na espécie canina, com cerca de 6,8\% de machos acometidos (YATES et al., 2003). Entretanto, em gatos a prevalência de criptorquidismo unilateral varia de 1,3 a 1,7\% (MILLIS et al., 1992; YATES et al., 2003).

\section{RELATO DE CASO}

Foi encaminhado para atendimento pelo Serviço de Nefrologia e Urologia do centro de diagnóstico e especialidades Saúde e Ciência Animal (SCAN), Campinas (SP), Brasil, um gato macho da raça Maine Coon, de 1 ano e 4 meses, com 3,7 kg, com apatia e hiporexia. A tutora relatou que, quatro dias antes, o animal havia passado por orquiectomia unilateral direita, pois o outro testículo não fora localizado. O paciente estava recebendo cefalexina $(30 \mathrm{mg} / \mathrm{kg}$ BID) e dipirona (25 mg/kg BID) por via oral desde o procedimento cirúrgico. Como histórico, o animal havia sido comprado filhote e sempre fora um animal magro, com apetite seletivo e urinava somente uma vez ao dia, sem outras queixas. A primeira ultrassonografia abdominal, solicitada após procedimento cirúrgico, mostrou que o rim direito media 6,1 cm (enquanto o esquerdo media 5,5 cm), apresentava adelgaçamento da cortical e dilatação acentuada da pelve, sugerindo hidronefrose (Figura 1A). Ainda, o testículo esquerdo apresentava-se em região hipogástrica média, com aspecto, ecotextura de parênquima e contornos sem alterações, medindo 0,9 ×0,4 cm (Figura 1B). 

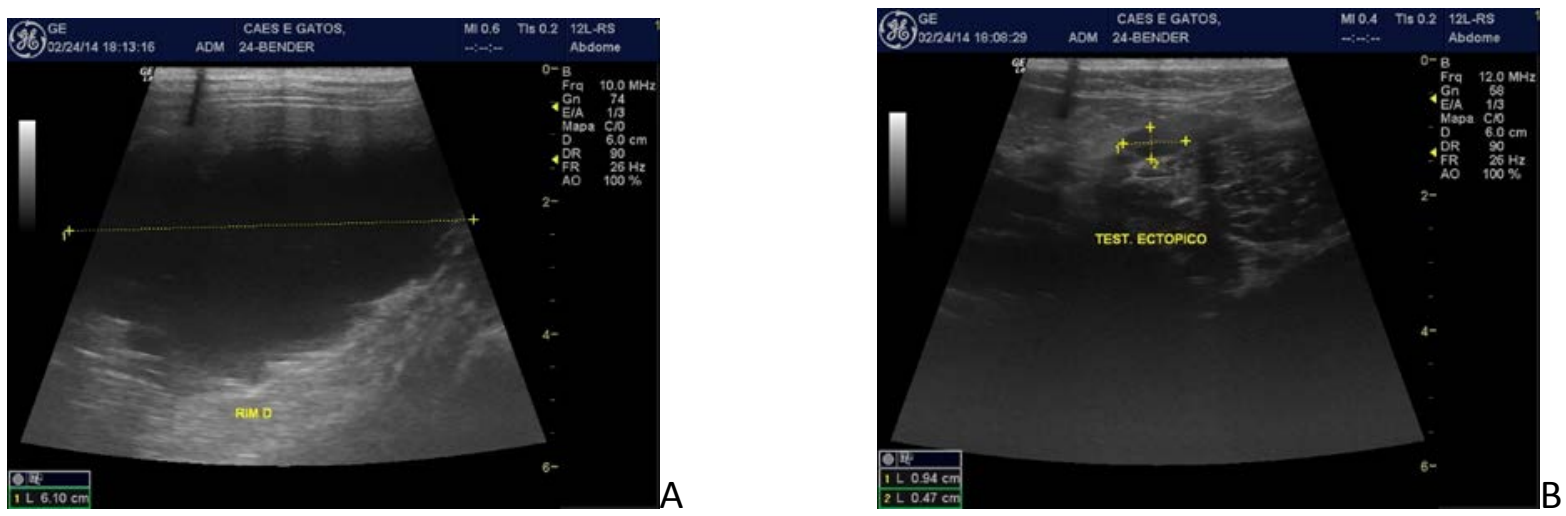

Figura 1 - Imagens ultrassonográficas evidenciando rim direito com perda total de parênquima ao ultrassom (A) e testículo esquerdo em região hipogástrica (B).

À inspeção visual, o animal estava taquipneico e apático. Ao exame físico, apresentava escore de condição corporal 2 (de 1 a 5), edema e dor na ferida cirúrgica, o que impossibilitou a palpação abdominal. Foi solicitado novo ultrassom, que confirmou os achados ultrassonográficos anteriores e ainda revelou hidroureter direito em toda a sua extensão até a região dorsocaudal à vesícula urinária, com desembocadura caudal ao trígono vesical, trajeto tortuoso em sua porção proximal, cuja mensuração foi de $0,5 \mathrm{~cm}$ de diâmetro em terço proximal e médio e $0,9 \mathrm{~cm}$ em terço distal, compatível com ureter direito ectópico. Além disso, notou-se discreta dilatação da pelve do rim esquerdo $(0,3 \mathrm{~cm})$, vesícula urinária com repleção acentuada e sem alterações, e testículo esquerdo localizado em região inguinal esquerda a $1,2 \mathrm{~cm}$ de profundidade em relação ao tecido subcutâneo. Os exames laboratoriais mostraram hemograma sem alterações, azotemia (creatinina sérica de 2,1 $\mathrm{mg} / \mathrm{dL}$ ) e hipercalemia (5,6 mEq/L). Na urinálise, observou-se densidade urinária maior que 1,040, pH 6 e sedimento inativo. Foram feitos também mensuração da pressão arterial e eletrocardiograma, sem alterações dignas de nota.

O animal foi encaminhado para novo procedimento cirúrgico no Hospital Veterinário Verlengia, Campinas (SP), para nefroureterectomia total direita (Figura 2A) e orquiectomia do testículo ectópico. Observou-se no transcirúrgico que o ureter esquerdo também apresentava dilatação, porém mais discreta que do ureter direito e sua inserção ocorria na 
uretra na mesma altura do ureter ipsilateral, de forma extraluminal (Figura 2B). Foi necessário também fazer desbridamento da ferida cirúrgica anterior, devido à presença vários abscessos subcutâneos. Optou-se por não fazer ureteroneocistostomia esquerda naquele momento e o paciente seria submetido a novo procedimento cirúrgico após recuperação. O animal permaneceu internado e recebeu fluidoterapia de manutenção com solução fisiológica a 0,9\% por via IV a $8 \mathrm{ml} / \mathrm{kg} / \mathrm{h}$, cloridrato de tramadol $(2,0 \mathrm{mg} / \mathrm{kg}$ TID $/ \mathrm{SC})$, meloxicam $(0,05 \mathrm{mg} / \mathrm{kg} \mathrm{BID} / \mathrm{SC})$ e cefovecina sódica $(8,0 \mathrm{mg} / \mathrm{kg}$ em dose única/SC). Cerca de 10 horas após o procedimento cirúrgico, o paciente apresentou hipotensão e, mesmo após restabelecimento da pressão arterial, veio a óbito. A tutora não autorizou a necropsia do paciente para que se pudesse estabelecer a causa definitiva da morte.
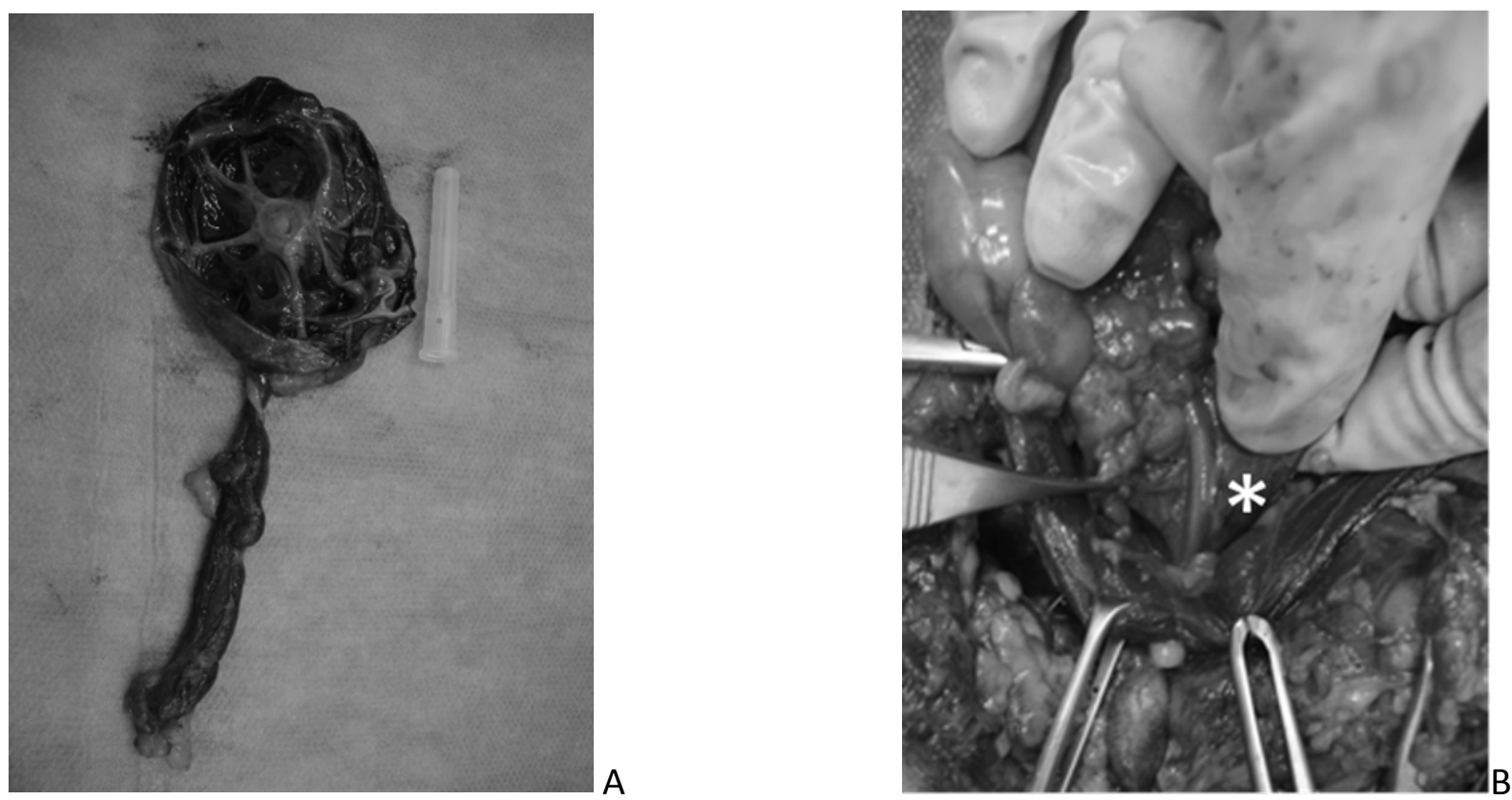

Figura 2 - (A) Rim e ureter direitos, evidenciando perda dos tecidos renais medular e cortical. (B) Asterisco indicando inserção do ureter esquerdo caudal ao trígono vesical.

\section{RESULTADOS E DISCUSSÃO}

Este é o segundo caso de ectopia bilateral de ureteres extramurais em gato da raça Maine Coon. O sinal clínico comumente apresentado é a incontinência urinária desde o nascimento (BIEWENGA et al., 1978; CRIVELENTI et al., 2013; KUZMA; HOLMBERG, 1988). De acordo com 
Macphail (2014), quando a abertura do ureter é perto do trígono vesical, o animal pode não ter nenhum sinal clínico específico, como a incontinência urinária, caso deste paciente. Pelo fato de os dois ureteres serem ectópicos, a vesícula urinária apresentar-se repleta ao ultrassom e o animal apresentar comportamento miccional normal, pode-se inferir que a urina refluía da uretra para a vesícula urinária para sua distensão.

A literatura cita que vários métodos de imagem podem ser utilizados para o diagnóstico, como ultrassonografia, urografia excretora e cistoscopia (HECHT; HENRY, 2011). O diagnóstico pode ser feito pelo ultrassom quando o ureter dilatado pode ser acompanhado passando pelo trígono vesical e desembocando caudalmente a essa estrutura. Entretanto, quando a dilatação é mínima, associada a transdutor de baixa frequência ou obesidade do paciente, a visualização deste órgão torna-se extremamente difícil. Ainda, as junções ureterovesicais são pequenas estruturas que podem ser difíceis de identificar e o refluxo da uretra para a vesícula urinária pode ser confundido com jato ureteral (LAMB, 1998). Exames laboratoriais não mostram alterações até que a hidronefrose promova perda funcional do parênquima renal ou quando ocorre pielite ou pielonefrite (HOELZLER; LIDBETTER, 2004). Este animal apresentou azotemia leve, mesmo com perda total de função do rim direito, e não teve alterações no hemograma. A urinálise, mostrando boa concentração urinária, revelou que, apesar da hidronefrose direita acentuada, o rim esquerdo mantinha sua função de forma adequada.

O tratamento baseia-se em ureteroneocistostomia (KUZMA; HOLMBERG, 1988) e, em casos em que há perda total de parênquima por hidronefrose, indica-se a nefroureterectomia total (MCLOUGHLIN; CHEW, 2000), procedimento este realizado no paciente relatado.

Testículos escrotais não são facilmente palpáveis em gatos filhotes e o diagnóstico do criptorquidismo é feito, normalmente, em gatos jovens antes de um ano de idade, quando da ocasião da orquiectomia eletiva (MEYERS-WALLEN, 2009), da mesma forma que ocorreu neste caso. Apesar de a raça Persa ser a mais acometida (YATES et al., 2003), o paciente em questão, apresentando simultaneamente duas patologias de desenvolvimento, era um Maine Coon. De acordo com Little (2011), a apresentação mais comum é a monorquidia 
unilateral inguinal. Neste caso, o paciente era criptorquida, com localização testicular intraabdominal.

\section{CONCLUSÃO}

Embora a ocorrência de ureteres ectópicos seja bastante incomum em gatos, este é o segundo relato na raça Maine Coon, em que ambos os casos foram relatados no Brasil. Ainda, a presença conjunta de criptorquidismo, outra anormalidade infrequente na espécie felina, chama a atenção para o diagnóstico precoce dessas alterações anatômicas.

\section{GENITURINARY TERATOGENESIS IN MAINE COON CAT}

\section{ABSTRACT}

$\mathrm{O}$ ccurrence of ectopic ureters is infrequent and mostly observed in bitches, which have urinary incontinence since young age. Cats are rarely affected by these two developmental abnormalities. This report aims to present the case of a male Maine Coon cat, with one year and four months of age, diagnosed with bilateral ectopic ureters, unilateral hydronephrosis, and unilateral cryptorchidism. The patient was referred by a colleague due to apathy and appetite loss after unilateral orchiectomy a few days before and did not have urinary incontinence. The ectopic ureter, right hydronephrosis and left ectopic testis were observed by ultrasound, while the left ectopic ureter was diagnosed during the surgery. Full right nephroureterectomy and left orchiectomy at that time were chosen, in addition to surgical wound debridement due to numerous subcutaneous abscesses from the first surgery performed. However, 10 hours after surgery the patient had hypotension and even after restoration of blood pressure, it came to death. The owner did not authorize necropsy, so the definite cause of death was not established.

Keywords: Ectopic. Feline. Hydronephrosis. Testicle. Ureter.

\section{TERATOGÉNESIS GENITURINARIA EN GATO MAINE COON}

\section{RESUMEN}

os relatos de uréteres ectópicos son poco frecuentes y más observadas en perras, que presentan incontinencia urinaria desde cachorras. Los gatos son raramente afectados - por esas dos anormalidades del desarrollo. Este relato tiene como finalidad presentar el caso de un gato macho Maine Coon, de un año y cuatro meses, con diagnóstico de uréteres 
ectópicos bilaterales, hidronefrosis unilateral, aparte de criptorquidismo unilateral. El paciente fue encaminado por un colega, debido a que presentaba apatía e hiporexía después de la orquiectomía unilateral realizada algunos días antes y no mostraba incontinencia urinaria. El uréter ectópico y la hidronefrosis derecha, así como el testículo ectópico izquierdo, fueron observados por medio de ultrasonografía, y la ectopía del uréter izquierdo fue observada durante el periodo transquirúrgico. Se optó por hacer la nefroureterectomía total derecha y la orquiectomía izquierda en aquel momento, fuera del desbridamiento de la herida quirúrgica debido a inúmeros abscesos subcutáneos provenientes de la primera cirugía realizada. Entretanto, 10 horas después de la cirugía el paciente tuvo hipotensión y, a pesar del restablecimiento de la presión arterial, el paciente falleció. La tutora no autorizó la realización de necropsia para que fuese posible investigar la causa de muerte definitiva.

Palabras clave: Ectopía. Felino. Hidronefrosis. Testículo. Ureter.

\section{REFERÊNCIAS}

BIEWENGA, W. J.; ROTHUIZEN, J.; VOORHOUT, G. Ectopic ureters in the cat - a report of two cases. Journal of Small Animal Practice, v. 19, n. 9, p. 531-537, 1978.

CRIVELENTI, L. Z.; MEIRELLES, A. E. W. B.; RONDELLI, M. C. H.; et al. Bilateral extraluminal ectopic ureters in a Maine Coon cat. Arquivo Brasileiro de Medicina Veterinária e Zootecnia, v. 65, n, 3, p. 627-630, 2013.

HECHT, S.; HENRY, G. A. Ultrasonography of the urinary tract. In: BARTGES, J.; POLZIN, D. Nephrology and Urology of Small Animals. Danvers: Wiley-Blackwell, 2011. Cap. 16, p. 128145.

HOELZLER, M. G.; LIDBETTER, D. A. Surgical management of urinary incontinence. Veterinary Clinics of North America: Small Animal Practice, v. 34, n. 4, p. 1057-1073, 2004.

KUZMA, A. B.; HOLMBERG, D. L. Ectopic ureter in a cat. Canadian Veterinary Journal, v. 28, p. 59-61, 1988.

LAMB, C. R. Ultrasonography of the ureters. Veterinary Clinics of North America: Small Animal Practice, v. 28, n. 4, p. 823-848, 1998.

LITTLE, S. Feline Reproduction: Problems and clinical challenges. Journal of Feline Medicine and Surgery, v. 13, p. 508-515, 2011.

MACPHAIL, C. M. Cirurgia de rim e ureter. In: FOSSUM, T. W. Cirurgia de Pequenos Animais. 4. ed. Rio de Janeiro: Elsevier, 2014. P. 2002-2085.

MCLOUGHLIN, M. A.; CHEW, D. J. Diagnosis and surgical management of ectopic ureters. Clinical Techniques in Small Animal Practice, v. 15, n. 1, p. 17-24, 2000. 
MEYERS-WALLEN, V. N. Review and Update: Genomic and Molecular Advances in Sex Determination and Differentiation in Small Animals. Reproduction in Domestic Animals, v. 44, n. 2, p. 40-46, 2009.

MILLIS, D. L.; HAUPTMAN, J. G.; JOHNSON, C. A. Cryptorchidism and monorchidism in cats: 25 cases (1980-1989). Journal of the American Medical Association, v. 200, p. 1128-1130, 1992.

NEWMAN, S. J. O Sistema Urinário. In: ZACHARY, J. F.; MCGAVIN, M. D. Bases da Patologia Veterinária. Rio de Janeiro: Elsevier, 2013. Cap. 11, p. 592-692.

YATES, D.; HAYES, G.; HEFFERNAN, M.; et al. Incidence of cryptorchidism in dogs and cats. Veterinary Record, v. 152, p. 502-504, 2003.

Autor para correspondência: Marcy Lancia Pereira.

Coordenadoria Especial de Biociências e Saúde Única, CCR/UFSC Curitibanos - Av. Advogado Sebastião Calomeno, s/n (CEDUP), CEP 89520-000, São Francisco, Curitibanos-SC. marcy.pereira@ufsc.br 